Results Then, anasarca and nephrosis was improved and S-Cr was decreased to $1.20 \mathrm{mg} / \mathrm{dL}$ in proportion to the reduction in activated Th17 cells in PD.

Conclusions Although recent studies have begun to shed light on the role of IL-17 in the pathogenesis of SLE, there is no convincing evidence in actual patients. In this case, improvement of disease activity of SLE was correlated with the decrease of activated Th17. This is the first report that the IL-17-targeted therapy for SLE was shown to be effective in a patient skewing towards Th17-phenotype.

\section{DEVELOPMENT OF ARTEMISININ ANALOGUEANALOG SM934 IN THE TREATMENT OF SYSTEMIC LUPUS ERYTHEMATOSUS}

J Zuo, W Tang*. Shanghai Institute of Materia Medica- Chinese Academy of Sciences, Immunopharmacology, Shanghai, China

\subsection{6/lupus-2017-000215.111}

Background and aims Besides their outstanding antimalarial activity, artemisinin and its derivatives also possess immunosuppressive activities and are clinical used to treat SLE. $\beta$-aminoarteether maleate (SM934), a water soluble artemisinin derivative, got the approval documents authorised by Chinese FDA for clinical trials. This study investigated the curative effects of SM934 on lupus-prone mice and explored its underlying therapeutic mechanisms.

Methods In vivo, SM934 was given orally to female NZB/W F1 and MRL/lpr mice; renal injury, peripheral lymphoid organ disease and serological changes were evaluated. Meanwhile, correlative pathological mechanisms were studied using different aged mice. Besides, the effects of SM934 on human PBMCs were also assessed.

Results We demonstrated that SM934 treatment could significantly improve SLE syndrome in lupus-prone animal models, including delayed the progression of glomerulonephritis; ameliorated proteinuria and renal lesion severity; increased the survival rate; decreased levels of BUN and serum anti-doublestranded DNA antibodies. Furthermore, clinical improvement was accompanied with decreased Th1-related anti- dsDNA IgG2a and IgG3 Abs, serum IL-17, and increased Th2-related anti-dsDNA IgG1 Ab, serum IL-10 and IL-4. Moreover, SM934 could significantly inhibit both of Th1 and Th17 responses, elevate Treg percentage and lower the percentage of $\mathrm{CD}^{+} \mathrm{B}^{+} 20^{+} \mathrm{CD}^{-} \mathrm{CD}^{-}$(double negative) $\mathrm{T}$ cells in $\mathrm{MRL} /$ lpr mice. We further elucidate that SM934 treatment restored the compartment of $\mathrm{B}$ cells in the spleen of $\mathrm{MRL} / \mathrm{lpr}$ mice by increasing quiescent $\mathrm{B}$ cells, maintaining germinal centre B cells, decreasing activated B cells and reducing PCs.

Conclusions This work provides new evidence and clues for research about artemisinin compounds in the field of autoimmune diseases.

\section{Other basic science}

\section{INCREASED NEUTROPHILS AND NEUTROPHIL SERINE PROTEASES IN THE SPLEENS OF ESTROGEN-TREATED C57BL/6 MICE AND IN SEVERAL STRAINS OF SPONTANEOUS LUPUS-PRONE MICE}

${ }^{1} \mathrm{R}$ Dai, ${ }^{1} \mathrm{C}$ Cowan, ${ }^{1} \mathrm{~B}$ Heid, ${ }^{1} \mathrm{D}$ Khan, 'Z Liang, ${ }^{2}$ CTN Pham, ${ }^{1}$ SA Ahmed*. 'VirginiaMaryland College of Veterinary Medicine- Virginia Tech, Biomedical sciences, Blacksburg, USA; ${ }^{2}$ Washington University School of Medicine, Department of Medicine- Division of Rheumatology, St. Louis, USA

\subsection{6/lupus-2017-000215.112}

Background and aims Oestrogen, a natural immunomodulator, regulates the development and function of diverse immune cell types and has been implicated in lupus development.

Methods To determine the regulatory role of oestrogen on neutrophil development and function, we treated B6 mice with placebo- or oestrogen implants for 6-8 weeks, and then analysed splenic neutrophil serine proteases (NSPs, such as

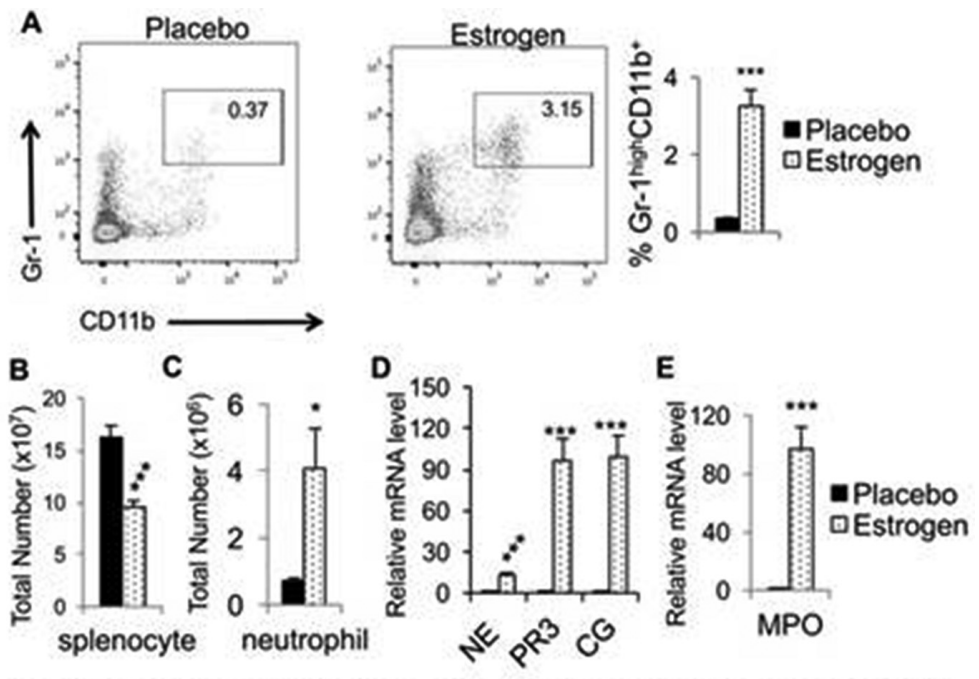

Abstract 112 Figure 1 Estrogen treatment increasesneutrophil number, NSP and MPO expression in wild type B6 mice. (A) Flow cytometry analysis of splenic neutrophil percentage in placebo-and estrogen-treated B6 mice. (B)The total splenocytes count in placebo- and estrogen-treated mice. (C) The total $C D 11 b^{*} G R 1^{*}$ splenic neutrophilcounts in placebo- and estrogen-treated mice. (D and E) Real-time RT-PCR analysis the expressoin of NSPs (D), and MPO (E) in splenocytes from placebo- and estrogen- treated mice. The graphs show means \pm SEMs ( $\mathrm{n} \geq 4$ ). Unpaired students ttests (placebo vs estrogen) were performed. ${ }^{*}, p<0.05 ;{ }^{* *}, p<0.01$; and ${ }^{* * *}, p<0.001$. 
A
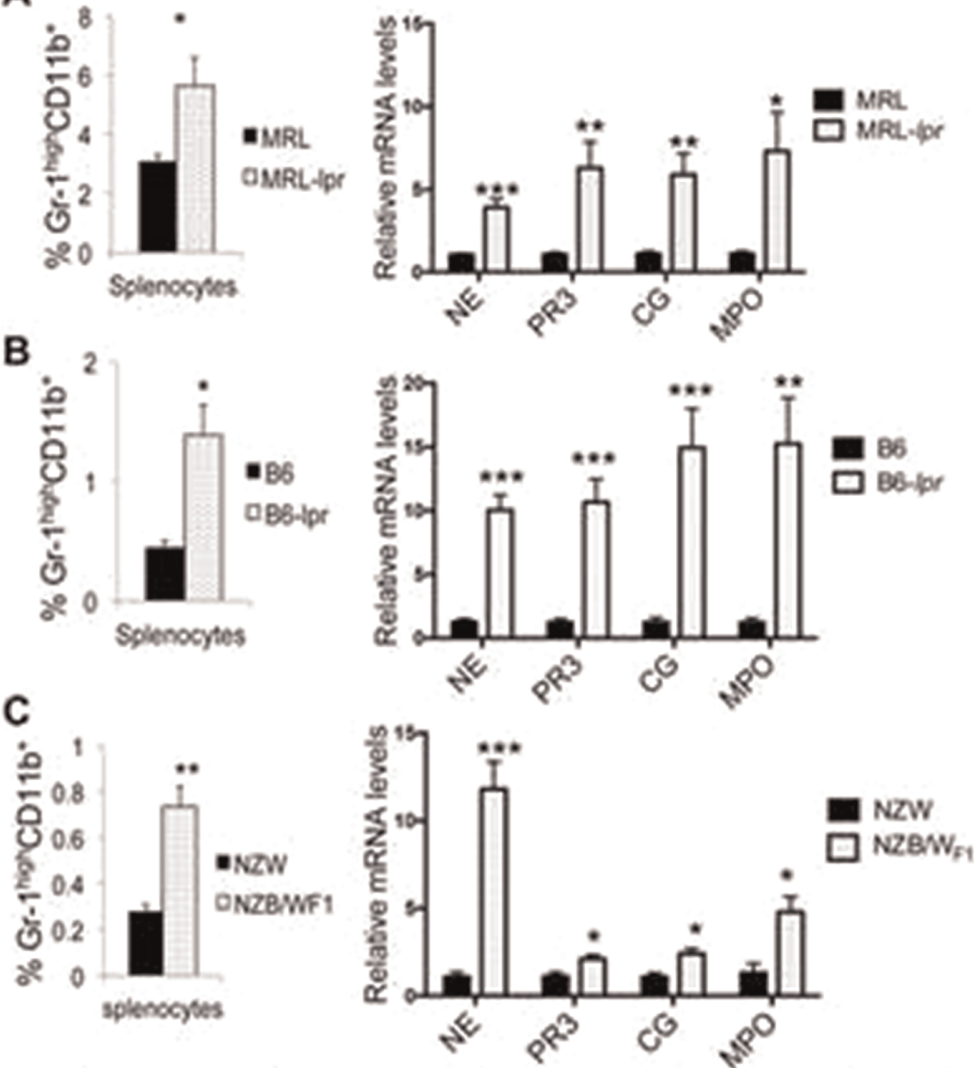

Abstract 112 Figure 2 Increased neutrophil percentages, NSPs and MPO expression in the spleen cells of three different murmine lupus strains.

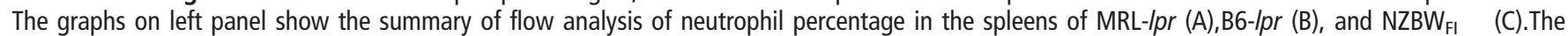
graphs on right panel summarized the real-time RT-PCR analysis of the relative expression levels of NE, PR3, CG, and MPO in the splenocytes of
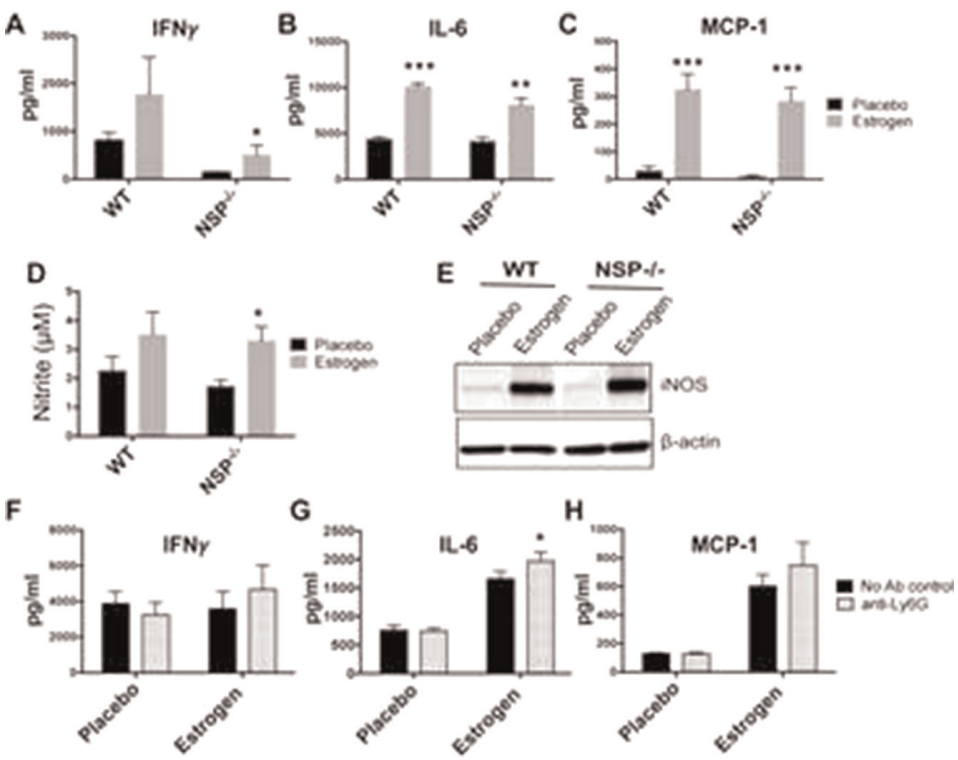

Abstract 112 Figure 3 Either depletion of NSPs in vivo or depletion splenic neutrophil in vitro has obvious effect on estrogen-mediated

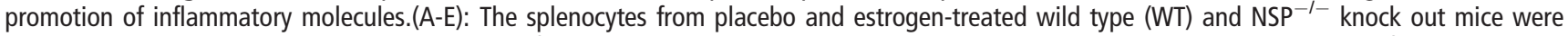
simulated with LPS to measure the production of IFN $\gamma$ (A, LPS 6 hours ), IL-6 (B, LPS 24hours), MCP-1(C, LPS 24 hours), and inflammatory molecule NO (D,LPS 48 hours). The expression iNOS protein levels in activated splenocytes (LPS placebo-and estrogen treated B6 mice were treated with antimouse Ly6G antibody to deplete neutrophils, and then simulated with LPS for 24 hours to measure IFN $\gamma(\mathrm{F})$, IL-6 (G), and MCP-1 (H) production.The graphs show means \pm SEMs $\left(\mathrm{n} \geq 3\right.$ ). Unpaired student $t$ tests (No Ab vs anti-Ly6G) were performed; ${ }^{*}, p<0.05 ;{ }^{* *}, p<0.01 ;$ and ${ }^{* * *}, p<0.001$. 
neutrophil elastase (NE), proteinase 3 (PR3), and cathepsin G (CG)) and myeloperoxidase (MPO) expression by qRT-PCR. $\mathrm{NE}^{-/} / \mathrm{PR}^{-/} / \mathrm{CG}^{-/-}$triple knockout mice and in vitro depletion of neutrophils approaches were performed to determine the role of NSPs and neutrophils in estrogen-mediated inflammatory responses. The splenic neutrophil and NSPs expression in lupus-prone MRL-lpr, $\mathrm{B} 6-l p r$ and $\mathrm{NZB} / \mathrm{W}_{\mathrm{F} 1}$ and their respective controls were also analysed.

Results Although oestrogen reduced total splenocytes number, it markedly increased the splenic neutrophil numbers, NSPs and MPO expression in B6 mice (Figure 1). Splenic neutrophils, NSPs and MPO were also significantly increased in MRL-lpr, B6-lpr and NZB/ $/ \mathrm{W}_{\mathrm{F} 1}$ mice (Figure 2). Despite of the critical role of NSPs and neutrophils in inflammation, depletion of NSPs in vivo did neither affect oestrogen's ability to increase in splenic neutrophils nor the induction of inflammatory mediators from ex vivo activated splenocytes, and depletion of splenic neutrophils in vitro had also no obvious effect on NSPs expression (due to the increase of NSPs in cells other than neutrophils) and LPS-induced IFNg and MCP-1 (Figure 3).

Conclusions Overall, we demonstrated a remarkable commonality with regards to the increase of neutrophils and NSPs in the spleens of autoimmune-prone mice and estrogen-treated B6 mice.

\section{EFFECT OF MICROPARTICLES DERIVED FROM PATIENTS WITH LUPUS ERYTHEMATOSUS SYSTEMIC (SLE) ON MODULATION OF MICRORNAS 146A AND 126, IN A MONOCYTE CELL LINE}

${ }^{1} \mathrm{G}$ Vasquez, ${ }^{1} \mathrm{U}$ Carmona-Perez ${ }^{*},{ }^{2} \mathrm{CH}$ Muñoz-Vahos, ${ }^{2} \mathrm{AL}$ Vanegas-Garcia, ${ }^{1} \mathrm{M}$ Rojas. ${ }^{1}$ Universidad de Antioquia, Grupo de Inmunología Celular e Inmunogenética GICIG. Instituto de Investigaciones Médicas- Facultad de Medicina-, Medellin, Colombia; ${ }^{2}$ Hospital Universitario San Vicente Fundación, Sección Reumatología, Medellin, Colombia

\subsection{6/lupus-2017-000215.113}

Background and aims Important miRNAs are involved in the modulation of immune functions and can be found in the intra- and extracellular environments, in circulation attached to RNA-binding molecules or packed in form of microparticles (MP). Given this, MP could have an important role in intercellular communication, modulation and expression of miRNAs in their target cells.

To establish whether MP of SLE patients can modulate the expression of miRNAs (miRNA 126, miRNA 146a) and their target molecule (interferon response factor 5, IRF5) in the monocyte cell line U937.

Methods MP obtained from serum of SLE and other autoinmune diseases (OAD) patients, and healthy controls were used as stimulus in the cell line U937 to evaluate their effect over: 1) The expression of membrane markers through flow cytometry, 2) content of miRNAs 126 and 146a through PCR and 3) expression of their target molecule, IRF5 by Western Blot. Results We observed, a decrease in HLA-DR, CD18, CD119 and an increase of IL-6 in U937 stimulated with MP from healthy controls, patients with active and inactive SLE, as well as patients with OAD. Additionally, a positive effect over the expression of miR126 and a negative effect over the expression of miR146a were observed. IRF5 as a target of miRNA146, did not change after MP treatment independent of MP origin.
Conclusions Our results suggested that MPs may have a regulatory effects, inducing a decreased expression of membrane molecules and miRNAs-146 levels, without effect in IRF5. In addition, MPs increased levels of cytokines and miRNA-126, latter is related with the demethylation.

\section{N-ACETYL-L-CYSTEINE (NAC) CONTROLS OSTEOCLASTOGENESIS THROUGH REGULATING TH17 DIFFERENTIATION AND RANKL PRODUCTION IN RHEUMATOID ARTHRITIS}

${ }^{1} \mathrm{HR}$ Kim*, ${ }^{2} \mathrm{BM}$ Kim, ${ }^{1} \mathrm{KA}$ Lee, ${ }^{1} \mathrm{SH}$ Lee, ${ }^{2} \mathrm{KW}$ Kim. ${ }^{1}$ Konkuk University Hospital, Rheumatology, Seoul, Republic of Korea; ${ }^{2}$ Seoul St. Mary's Hospital- College of MedicineThe Catholic University, Convergent Research Consortium for Immunologic disease, Seoul, Republic of Korea

\subsection{6/lupus-2017-000215.114}

Background and aims This study aimed to determine the regulatory role of N-Acetyl-l-cysteine (NAC), an antioxidant, in IL17-induced osteoclast differentiation in rheumatoid arthritis (RA).

Methods After RA synovial fibroblasts were stimulated by IL17 , the expression and production of RANKL was determined by real-time PCR and ELISA. Human peripheral blood monocytes were cultured with M-CSF, IL-17, RANKL, and/or various concentrations of NAC, followed by counting of the cells for tartrate-resistant acid phosphatase activity to determine osteoclast formation. Osteoclastogenesis was also determined after cocultures of IL-17-stimulated RA synovial fibroblasts, Th17 cells and various concentrations of NAC with monocytes. After human peripheral $\mathrm{CD}^{+} \mathrm{T}$ cells were cultured with NAC under Th17 condition, IL-17, IFN-g, IL-4, Foxp3, RANKL and IL-2 expression and production was determined by flow cytometry or ELISA.

Results When RA synovial fibroblasts were stimulated by IL17, IL-17 stimulated the production of RANKL, and NAC reduced the IL-17-induced RANKL production in a dosedependent manner. NAC decreased IL-17-activated phosphorylation of mTOR, JNK and IkB. When human peripheral blood $\mathrm{CD}_{14}{ }^{+}$monocytes were cultured with M-CSF and IL17 or RANKL, osteoclasts were differentiated, and NAC reduced the osteoclastogenesis. After human peripheral CD4 ${ }^{+}$ $\mathrm{T}$ cells were co-cultured with IL-17-pretreated RA synovial fibroblasts or Th17 cells, NAC reduced their osteoclastogenesis. Under Th17 polarising condition, NAC decreased Th17 cell differentiation and IL-17 and RANKL production.

Conclusions NAC inhibits the IL-17-induced RANKL production in RA synovial fibroblasts and IL-17-induced osteoclast differentiation. NAC also reduced Th17 polarisation. NAC could be a supplementary therapeutic option for inflammatory and bony destructive processes in RA.

\section{QUNANTITATIVE AND FUNCTIONAL EVALUATION OF PLASMA MICROPARTICLES IN SYSTEMIC LUPUS ERYTHEMATOSUS}

\begin{abstract}
${ }^{1,2}$ A Lateef* ${ }^{*}{ }^{1} \mathrm{~L}$ Shaffiee, ${ }^{3} \mathrm{GK}$ Gill, ${ }^{1} \mathrm{P}$ Cheung, ${ }^{4} \mathrm{YC}$ Lim. ${ }^{1}$ National University Hospital, Medicine, Singapore, Singapore; ${ }^{2}$ Yong Loo Lin School of Medicine- National University of Singapore, Medicine, Singapore, Singapore; ${ }^{3}$ National University of Singapore, Physiology, Singapore, Singapore; ${ }^{4}$ National University of Singapore, Pathology, Singapore, Singapore
\end{abstract}

10.1136/lupus-2017-000215.115 